\title{
INICIACIÓN TEMPRANA EN INVESTIGACIÓN. MODELOS INSTRUCCIONALES ACTUALES
}

\author{
Arturo Silva Rodríguez y Esperanza Guarneros Reyes \\ Universidad Nacional Autónoma de México \\ México
}

\begin{abstract}
RESUMEN
En este artículo se analiza la manera en que se ha querido establecer en el estudiante universitario las competencias iniciales de investigación. La atención se enfoca en las diferentes tendencias que se han seguido para enseñar tempranamente al estudiante a investigar. Para hacer lo anterior, en primer lugar, se aborda la función de la universidad en la iniciación temprana a la investigación y después se presentan las tendencias que se han seguido en el pregrado para impulsar la formación inicial de los estudiantes para la investigación. Dichas tendencias se engloban en cinco modelos instruccionales: 1) demostrativo; 2) procedimental; 3) transcriptivo; 4) computacional, y (5) integral. El artículo finaliza examinando de las bondades y limitaciones de cada uno de dichos modelos, y concluye que para impulsar de modo efectivo la iniciación temprana a la investigación es necesario diseñar espacios curriculares en los planes de estudio en el pregrado, considerando como punto de referencia un modelo instruccional integral que conjugue la reflexión teórica con el uso de metodologías variadas y se apliquen procedimientos cuantitativos y cualitativos para analizar la base empírica obtenida en el proceso de investigación.
\end{abstract}

\section{Palabras Clave:}

semilleros de investigación, métodos cuantitativos, estadística, investigación científica, modelo instruccional, modelo educativo.

\section{EARLY INITIATION IN RESEARCH. CURRENT INSTRUCTIONAL MODELS}

\begin{abstract}
This article discusses how initial research competencies have been seeking to be established in the college student. Atention focuses in different trends that have been followed toteach the student to investigate early. To do the above, first, the university's role in early initiation to research is addressed, and then the trends that have been followed are presented to boost the initial formation of students for research. Such trends are assembled in five instructional models: 1) demonstrative; 2) procedural; 3 ) transcriptive; 4) computational, and (5) integral. The article ends examining each models' benefits and limitations, and it concludes that to efectively boost early initiation to research, curricular spaces need to be designed in the undergraduate curriculum, considering as reference point an integral instructional model that conjures the theoretical reflection with the use of various methodologies and that cuantitative and cualitative procedures are applied to analyze the empirical basis obtained in the research proccess.
\end{abstract}

\section{Keywords:}

research seedbeds, quantitative methods, statistics, scientific research, instructional model, educative model

BitÁCORA DEL ARTículo:

| Recibido: 19 de Septiembre de 2019 | Aceptado: 23 Octubre de 2019 | Publicado en línea: Enero - Junio de 2020 | 


\title{
INICIACIÓN TEMPRANA EN INVESTIGACIÓN. MODELOS INSTRUCCIONALES ACTUALES
}

\author{
Arturo Silva Rodríguez y Esperanza Guarneros Reyes \\ Universidad Nacional Autónoma de México \\ México
}

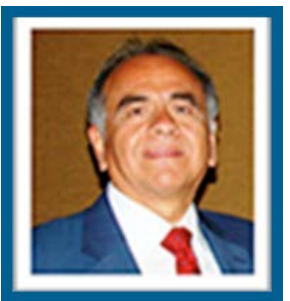

Arturo Silva Rodríguez

FES- Iztacala, UNAM

Correo: arturomeister@gmail.com

Doctor en Sociología por la Facultad de Ciencias Políticas y Sociales. Maestro en Modificación de Conducta. Especialidad en Matemáticas Aplicadas. Licenciatura en Psicología. Investigador responsable de varios proyectos relacionados con áreas de la psicología, las ciencias sociales en general, la educación y TIC.

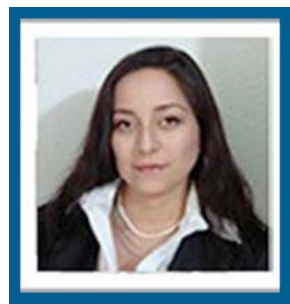

Esperanza Guarneros Reyes FES- Iztacala, UNAM

Correo:esperanzagr@gmail.com

Profesora de Carrera Asociada C Tiempo Completo de la Licenciatura de psicología en SUAyED y de la Maestría en Psicología en la FES Iztacala, UNAM. Pertenece al SNI. Responsable del Laboratorio Digital de Desarrollo Infantil LDDI de la FES Iztacala. Doctora en Psicología por la Facultad de Psicología UNAM,

\section{CONTRIBUCIÓN DE LOS AUTORES}

Esperanza Guarneros Reyes: Desarrolló el esquema básico del artículo y aportó ideas sobre el desarrollo de los modelos de enseñanza de las competencias iniciales de investigación | Arturo Silva Rodríguez: Desarrolló el esquema básico del artículo, así como la aportación de ideas para el desarrollo de las competencias iniciales de investigación, así mismo realizó la discusión y discusión del artículo.

\section{AGRADECIMIENTOS}

Se agredece al Proyecto financiado por PAPIME RR213918

\section{DATOS DE FiLIACIÓN DE LS Autores}

Facultad de Estudios Superiores Iztacala, UNAM

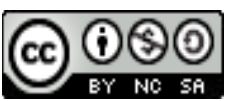

Copyright: (C) 2020 Silva-Rodríguez, A. \& Guarneros-Reyes, E.

Este es un artículo de acceso abierto distribuido bajo los términos de la licencia Creative Commons Reconocimiento-NoComercial 4.0 Internacional, por lo que su contenido gráfico y escrito se puede compartir, copiar y redistribuir total o parcialmente sin necesidad de permiso expreso de sus autoras con la única condición de que no se puede usar con fines directamente comerciales y los términos legales de cualquier trabajo derivado deben ser los mismos que se expresan en la presente declaración. La única condición es que se cite la fuente con referencia a la Revista Digital Internacional de Psicología y Ciencia Social y a sus autoras. 


\section{TABLA DE CONTENIDO}

LA UNIVERSIDAD Y SU FUNCIÓN EN LA INICIACIÓN TEMPRANA EN INVESTIGACIÓN

MODELOS INSTRUCCIONALES ACTUALES EN INICIACIÓN TEMPRANA EN INVESTIGACIÓN

1) Modelo instruccional demostrativo, 265

2) Modelo instruccional procedimental, 266

3) Modelo instruccional transcriptor, 267

4) Modelo instruccional computacional (o la "cultura digital del simple

o doble asterisco"), 270

5) Modelo instruccional integral, 272

Discusión 
$\mathbf{L}$ a investigación científica es uno de los principales motores para el desarrollo de cualquier sociedad. Su impacto se deja sentir en los más variados sectores sociales. La principal función es la generación de conocimiento que explique los fenómenos $y$, además, generar una tecnología que cambie el rumbo de los acontecimientos con la finalidad de lograr un mejor bienestar de los ciudadanos.

En las ciencias sociales y del comportamiento la investigación se practica cuando menos en tres grandes áreas. La primera está dirigida hacia la erudición que cultivan los círculos científicos que por lo general se sustenta en enfoques cuantitativos y en pocas ocasiones dirige su mirada hacia las aproximaciones cualitativas. En ésta se privilegia la teoría con la justificación de construir conocimiento que informe del fenómeno estudiado, con el firme propósito de encontrar el modelo que lo explique. Por su característica intrínseca textual se le ha denominado como una corriente discursiva que no se fundamenta en una agenda de investigación empírica (Rojek y Turner, 2000). Una segunda área en donde se practica es en el sector público y privado mediante centros de investigación independientes que por lo común se dedican desarrollar estudios relacionados con la evaluación de programas y políticas sociales, así como estudios de mercado. La tercera área es la vinculada con la manera en que se enseña a investigar en las universidades por medio de los planes de estudio. Esta área retoma la manera de investigar propia de los círculos científicos más interesados en la explicación teórica de los fenómenos, pero también incorpora aquella cuya finalidad es generar una tecnología social dirigida a cambiar el rumbo de dichos fenómenos. La perspectiva es incorporar en la enseñanza del proceso investigativo los escenarios más teóricos y especulativos, así como los entornos más aplicados.

En general, en el ámbito educativo universitario estas áreas de investigación son incluidas en los estudios de pregrado y posgrado; en los primeros en las asignaturas de metodología científica y estadística, y en los segundos como competencias específicas y generales que dotan al estudiante de conocimientos, habilidades, actitudes y valores para el desarrollo de investigación científica original de alta calidad en el ejercicio académico y profesional.
Por lo común los planes de estudio en el pregrado de las ciencias sociales y del comportamiento declaran que uno de sus objetivos es establecer en el estudiante competencias básicas de investigación. En general esta intención en la formación universitaria inicial se encomienda a las asignaturas relacionadas con la metodología científica y el análisis cuantitativo; recientemente se ha incorporado el análisis cualitativo como una competencia más que el estudiante debe tener (Immekus, 2019).

En este artículo se analiza la manera en que se ha pretendido establecer en el estudiante universitario las competencias iniciales de investigación. La atención se enfoca en las diferentes tendencias que se han seguido para enseñar tempranamente al estudiante a investigar, con la intención de que él mismo se vea como científico y que a que adquiera las competencias que le permitan comprender los hallazgos hechos al efectuar la investigación y sea capaz de generar y aplicar estrategias que produzcan cambios en los fenómenos estudiados. Para hacer lo anterior, en primer lugar, se aborda la función de la universidad en la iniciación temprana a la investigación y después se presentan las tendencias que se han seguido en el pregrado para impulsar la formación inicial de los estudiantes para la investigación. Dichas tendencias se engloban en cinco modelos instruccionales: 1) demostrativo; 2) procedimental; 3) transcriptivo; 4) computacional, y 5) integral. El artículo finaliza analizando las bondades y limitaciones de cada uno de dichos modelos instruccionales, y concluye que para impulsar de modo efectivo la iniciación temprana a la investigación es necesario impulsar en las universidades un modelo instruccional integral que conjugue la reflexión teórica con el uso de metodologías variadas y se apliquen procedimientos cuantitativos y cualitativos para analizar la base empírica obtenida en el proceso de investigación.

\section{LA UNIVERSIDAD Y SU FUNCIÓN EN LA INICIACIÓN}

\section{TEMPRANA EN INVESTIGACIÓN}

Corresponde a las universidades y a los institutos de investigación no sólo formar a las nuevas generaciones de profesionales que ofrezcan sus servicios a la sociedad, sino también establecer habilidades y competencias de investigación científica en el estudiante que lo capaciten para efectuar estudios inéditos y originales. Hoy existe en la sociedad la necesidad de formar investigadores jóvenes que rompan paradigmas y fronteras del conocimiento que les permitan transitar libremente entre las diferentes disciplinas agrupadas en el gran conglomerado de conocimientos científicos. La manera óptima de lograr este tránsito es adoptar una perspectiva trans- 
disciplinaria en la formación universitaria que tenga en la investigación científica su barco para navegar en los mares turbulentos formados por las fronteras de conocimiento de las diferentes disciplinas agrupadas alrededor de las ciencias sociales y del comportamiento.

Por tal motivo es imperioso que en las universidades se fomente, por medio del currículo formativo, el espíritu científico, en donde la investigación se presente como un proceso de descubrimiento creativo y conceptual que implica la formulación de preguntas correctas que se deben contestar. La investigación científica no es un proceso mecánico que sigue una fórmula de libro de cocina, como lo sugieren muchos manuales de investigación, algunos de los cuales se distribuyen libremente en Internet y otros constituyen la bibliografía básica en numerosos cursos de metodología científica y estadística de las variadas disciplinas que comprenden las ciencias sociales y del comportamiento. Por el contrario, es un proceso plagado de incertidumbre y contratiempos en el que se decide cómo proceder sobre la base de principios generales relacionados con una pregunta de investigación que se intenta responder.

Uno de los puntos más débiles en las universidades es la formación de investigadores en el pregrado, porque se parte de la premisa de que los estudios de doctorado son el espacio por excelencia para la enseñanza de la ciencia, y por ende para el establecimiento de competencias de investigación científica en el estudiante que le genere una actitud reflexiva y crítica que lo dirija a descubrir y crear nuevo conocimiento.

Cada vez más se manifiesta la necesidad de no sólo estimular desde el pregrado una actitud favorable hacia la ciencia, sino también instaurar un modelo educativo innovador que estimule desde el inicio de la formación universitaria el establecimiento en el estudiante de competencias para la investigación encaminadas a la generación de nuevos conocimientos y a la creación de tecnologías que mejoren el bienestar colectivo. Así, es necesario que la formación curricular para la investigación vaya más allá de cursos sueltos de metodología científica y de estadística que proliferan en la mayoría de las asignaturas que se imparten en el pregrado. Dichos cursos tienen un efecto muy reducido en el desarrollo conceptual y metodológico relacionado con la enseñanza y el aprendizaje para investigar (Guerrero-Useda, 2007) y los contenidos temáticos, los cuales, según opinión del estudiante, son fragmentados, descontextualizados, rígidos y con frecuencia incomprensibles y desmotivantes (Baum y Steele, 2017; Eudave Muñoz, Carvajal Ciprés, y Páez, 2018; Immekus, 2019; Jones, 2019; Roy, 2019).
A lo largo de los años estos cursos sólo han representado esfuerzos aislados que no responden a una política educativa clara en donde la universidad asuma el compromiso de hacer una innovación curricular encaminada a iniciar tempranamente al estudiante en investigación desde el pregrado. Una respuesta a esta falta de compromiso de formar de manera temprana para la investigación ha surgido la iniciativa en algunas universidades de Latinoamérica de crear semilleros (Cvetkovic-Vega, Inga-Berrospi y Mestas, 2017; Gómez-Restrepo, 2017; Guerra-Molina, 2017; Numa-Sanjuan y Márquez-Delgado, 2019; Rodríguez-Morales, Milanés-Gómez y Avila-Portuondo, 2016; Saavedra-Cantor, Antolínez-Figueroa, Puerto-Guerrero, Muñoz-Sánchez y Rubiano-Mesa, 2015; Tovar-Torres, 2018; Villalba-Cuéllar y González-Serrano, 2017; Zapata-Peña , Ortiz-Puerto, Bautista-Rincón, Díaz-González, Tinoco y Ayala-Cortés, 2014).

Al margen de los nombres que adopta esta iniciativa en las distintas regiones, lo importante es que todas ellas tienen como objetivo que el estudiante trabaje cerca de un profesor investigador o que se integre a un grupo de investigación para que aprenda a plantear problemas, formular hipótesis, recopilar información y sintetizarla, utilizar herramientas metodológicas para diseñar estudios de investigación, trabajar en equipo y discutir en grupo los alcances de los resultados, entre otras cosas.

Además de ser una innovación curricular en algunos estudios de pregrado, la iniciación temprana para la investigación es una respuesta de las universidades a la creciente demanda de la sociedad de que no sólo sean instituciones que formen profesionales, sino que en este mundo globalizado sean espacios en los que se forme a los jóvenes mediante la investigación en la ciencia y la tecnología, con la finalidad de que se apropien de los hechos reales y se enfrenten a los innumerables problemas que tiene que sortear la sociedad y que elija aqueلlión es importante para la humanidad. ${ }^{1}$ 1 Uno de estos esfuerzos de incluir de modo curricular la formación para la investigación a partir del pregrado, que funciona desde 2005, es la licenciatura en el Sistema Universidad Abierto y Educación a Distancia, conocido como SUAyED Psicología, que se imparte en la Universidad Nacional Autónoma de México (UNAM) desde la Facultad de Estudios Superiores Iztacala (FESI). Esta iniciativa fue uno de los primeros pasos que se dieron en la UNAM para estimular la producción científica desde los estudios de pregrado con el propósito de contribuir en la consolidación de la sociedad del conocimiento y la interacción, iniciando tempranamente al estudiante en el cultivo de la ciencia por medio de la investigación científica. La manera en que fue posible hacer esto se abordará en un siguiente artículo. 


\section{Modelos instruccionales actuales en}

\section{INICIACIÓN TEMPRANA EN INVESTIGACIÓN}

Las tendencias para formar tempranamente competencias de investigación en el estudiante de las ciencias sociales y del comportamiento han sido muy variadas; de manera tradicional las universidades han abordado el tema diseñando espacios curriculares en sus planes de estudio que están asociados con asignaturas de Matemáticas I, II, III, y en algunos casos hasta matemáticas IV; y por si no fuera suficiente, en pocas disciplinas de este conglomerado de ciencias hasta asignaturas de álgebra y cálculo, pero sobre todo asignaturas de metodología científica y de estadística que en ocasiones son bautizadas como métodos cuantitativos o procesos estadísticos, etcétera.

Los hechos que han perfilado los modelos instruccionales actuales han adoptado diferentes matices, pero todos podrían englobarse en cinco principales, considerando la manera en que fueron diseñados y planeados los cursos de metodología científica y del modo en que se analiza la información cuantitativa o cualitativa. Éstos podrían englobarse en cinco modelos instruccionales: 1) demostrativo; 2) procedimental; 3) transcriptivo; 4) computacional, y 5) integral. A continuación, se presentan y examinan las características de cada uno.

\section{1) Modelo instruccional demostrativo}

La característica principal del modelo instruccional demostrativo es el predominio del paradigma de las ciencias formales en la enseñanza temprana de investigación en el estudiante de pregrado. Es el más tradicional y se importó sobre todo de la enseñanza de las matemáticas. El modelo instruccional se enfoca en la declaración de variables abstractas sin ningún referente empírico, en la deducción y en el manejo de expresiones simbólicas. Por tanto, la clase se enfoca en la demostración rigurosa de teoremas como:

Mostrar que las varianzas del conjunto de $\mathrm{n}$ números

$$
a, a+d, a+2 d, \ldots, a+(n-1) d
$$

[es decir, una progresión aritmética con primer término a y razón d] está dada por

$$
1 / 12(n 2-1) d 2
$$

Y cuando se quiere enseñar la operatividad del cálculo de un procedimiento estadístico se utiliza la clásica y famosa declaración:

Sean dos variables continuas $X$ y $Y$

En seguida se presenta una lista de números sin ningún referente empírico de la metodología utilizada para recolectar esa información y se procede a hacer los cálculos para obtener el resultado correcto. Una clase de estos ejercicios es cuando se pide que de acuerdo con la tabla $X$ ajustar una recta de mínimos cuadrados utilizando a $X$ como la variable independiente y a $Y$ como la variable dependiente. Arreglo de números como el mostrado en la tabla 1 (tomada de Spiegel [1977] y referida como tabla 8-39) es por excelencia la manera didáctica como se presenta la información en la clase o en los libros de texto que se utilizan para enseñar la habilidad de cálculo al estudiante.

Tabla 1.

Tomada de Spiegel (1977) y referida como tabla 8-39.

\begin{tabular}{|l|l|l|l|l|l|l|}
\hline $\mathrm{x}$ & 2 & 4 & 5 & 6 & 8 & 11 \\
\hline $\mathrm{y}$ & 18 & 12 & 10 & 8 & 7 & 5 \\
\hline
\end{tabular}

En el plano pedagógico el modelo instruccional demostrativo de iniciación temprana en investigación le da prioridad a la comprobación del teorema y al algoritmo de cálculo, que la mayoría de las veces se hace de memoria y muy pocas veces se utiliza una calculadora personal, porque se cree que hacer los cálculos sin ninguna herramienta tecnológica contribuye a que el estudiante ejercite su mente, considerándose como una especie de gimnasia mental.

En cuanto a la técnica didáctica, ésta consiste en solicitar al estudiante hacer ejercicio tras ejercicio hasta que domine el algoritmo de cálculo de la técnica estadística de investigación que se esté enseñando. La dinámica del curso se enfoca en la formulación de modelos cuantitativos y en la demostración de las distintas fórmulas contenidas en el modelo.

En consecuencia, esta tendencia demostrativa se aleja de modo radical del proceso de investigación en las ciencias sociales y del comportamiento que va y viene entre el mundo abstracto y el mundo concreto, transitando desde el planteamiento del problema, diseño de recolección de información, análisis de dicha información y conclusiones. Este modelo instruccional olvida que la estadística es una disciplina metodológica que existe por sí misma, y al igual que muchas otras disciplinas utiliza el pensamiento y el lenguaje matemático sólo con fines de desarrollo; su fortaleza en el plano educativo consiste en que robustece la enseñanza, el aprendizaje y la comprensión del proceso de investigación científica (MacGillivray, 2019).

La estadística es una derivación de las matemáticas que gira alrededor de un tipo de pensamiento diferente al matemático, porque los datos no son sólo números; son números en un contexto y tienen una historia; coloquialmente se diría que tienen nombre y apellido, porque provienen de una operación metodológica de recolección empírica de información. 
Como se indicó, por su parte el modelo de diseño curricular está dominado —en algunas disciplinas de las ciencias sociales y del comportamiento- por la creación de asignaturas como Matemáticas I, II, III, y en algunos casos hasta matemáticas IV, y por si no fuera suficiente en pocas disciplinas de este conglomerado de ciencias hasta asignaturas de álgebra. Debido a la forma en que fueron concebidos, lo común en todos esos cursos es que la estrategia didáctica consiste en demostrar argumentos constituidos por secuencias lógicas de premisas, a la manera de axiomas, que desembocan en la declaración de una conclusión. Lo preocupante es que esta situación no es exclusiva en las ciencias sociales y del comportamiento, sino que - como señalan Zapata-Cardona y González-Gómez (2017) — es la manera en que se enseña tradicionalmente en los salones de clase del sistema educativo en general, en donde se privilegia la fundamentación matemática, las demostraciones deductivas y el manejo de expresiones simbólicas.

En esta tendencia, cómo olvidar los libros clásicos de la serie Shaum publicados por McGrawHill, que eran la fuente que alimentaron inicialmente esta tendencia. Constituyeron la bibliografía básica de la mayoría de los cursos de estadística —en algunos hoy aún lo son-, en los cuales se tenían expectativas de establecer en el estudiante las bases iniciales de algunas competencias de investigación científica. En ellos se encontraba una gran variedad de ejercicios que eran una fuente interminable que alimentaba la dinámica de la clase. Los libros Probabilidad y estadística y Estadística son una muestra fehaciente de ese modelo instruccional demostrativo con el que se pretendía establecer competencias de investigación científica en los alumnos que cursaban alguna disciplina de las ciencias sociales y del comportamiento.

\section{2) Modelo instruccional procedimental}

Este modelo instruccional de iniciación temprana en investigación se caracteriza por traducir la base conceptual y formal de las matemáticas que sostiene al análisis cuantitativo en la base empírica de las ciencias sociales y del comportamiento. La característica principal de esta tendencia consiste en presentar algunos algoritmos y después hacer los ejercicios de aplicación de dichos algoritmos. A diferencia de la tendencia demostrativa, la procedimental parte de la idea de que el contexto de donde se obtienen los datos y la historia de cómo se recolectan éstos hacen que el análisis cuantitativo, personificado más llanamente en la estadística, sea un área del conocimiento si no muy diferente, sí distinta a las matemáticas. La estadística proporciona un conjunto de ideas y herramientas para analizar los datos a otras disciplinas sobre todo empíricas, mientras que el interés de las matemáticas se enfoca en el descubrimiento de patrones e ideas abstractas que son únicas y universales obtenidas por deducción (Cobb y Moore, 1997).

Esto originó que, en la enseñanza del análisis cuantitativo en la investigación, y en particular en los cursos de estadística, se dirigiera a mostrar los referentes empíricos en que era factible aplicar tal o cual modelo matemático, con la premisa de que los datos no son sólo números, sino que son números en un contexto y que la estadística es el medio para unir los conceptos abstractos de las matemáticas con los datos recolectados en investigaciones empíricas.

Como consecuencia, en los cursos y en los libros de texto se hace a un lado la demostración de los algoritmos matemáticos y se hacen traducciones de éstos — con referentes empíricos - de las ciencias sociales y del comportamiento. La expresión "Sean dos variables continuas X y $Y^{\prime \prime}$ desaparece, y las incógnitas son sustituidas por el tiempo de duración de los focos, el índice de tornillos defectuosos, la venta promedio de carne roja de una cadena de supermercados, duración de los neumáticos, etcétera.

Fue sólo cuestión de tiempo que en las ciencias sociales y del comportamiento se adoptara esta práctica de transcribir los conceptos formales por la nominación de variables de las disciplinas de estas ciencias. El tiempo de duración de los focos se sustituyó por el tiempo en que un niño dedica a hacer una tarea; el índice de tornillos defectuosos por el índice de personas que tienen un empleo fijo, etcétera. De hecho, lo único que se hizo fue nominar y asignarles un apellido a las incógnitas $X$ y $Y$, apellidos que surgen de la jerga conceptual de las disciplinas que conforman las ciencias sociales y del comportamiento. De acuerdo con esta tendencia se publicaron libros que hacían referencia a la investigación, sobre todo cuantitativa, en las diversas disciplinas que conforman a dichas ciencias. Cómo no recordar libros como Métodos estadísticos aplicados a las ciencias sociales (de Glass y Stanley), Estadística social (de Blalock), Metodología y técnicas de la investigación en ciencias sociales (de Pardinas), Estadística para administración y economía (de Anderson, Sweeney y Williams), Estadística aplicada a los negocios y a la economía (de Wesbster), Análisis de series temporales económicas y Bioestadística (de Daniels), etcétera.

Si bien fueron esfuerzos encomiables que formaron generaciones de estudiantes, la línea editorial de esos libros se enfocaba sobre todo en la enseñanza de los conceptos lógicos-matemáticos que subyacen a las herramientas cuantitativas que sirven de sustento a las investigaciones en las ciencias sociales y del compor- 
tamiento, en lugar de enseñar el modo de aplicar esas mismas herramientas en el análisis de los estudios científicos que se desarrollan en esas ciencias. En consecuencia, la mayoría de los libros de esta tendencia se enfocan a establecer competencias relacionadas con los conocimientos declarativos, demostrativos y procedimentales de cómo calcular una determinada técnica estadística más que en establecer competencias reflexivas para aplicar y utilizar los conceptos lógico-matemáticos de las herramientas cuantitativas en los campos de investigación de dichas ciencias.

Derivado de esta situación, para establecer competencias de investigación en los estudiantes el modelo educativo continuó siendo mecanicista; en los espacios curriculares se redujo el tiempo de la demostración de los teoremas matemáticos y se invirtió más tiempo en enseñar paso a paso la manera de calcular una determinada técnica estadística.

Aún hoy, inicio de la tercera década del siglo XXI, se continúa con la publicación de libros que mantienen esta línea editorial, y lo que es más preocupante es que no enfoca en mostrar la manera de calcular determinada técnica estadística paso a paso sobre los datos ficticios que se le presentan, que culmina en una decisión simple e ingenua de aceptar una hipótesis nula o alterna. Esta tendencia procedimental es una imagen distorsionada y muy alejada de lo que en realidad es el proceso de investigación científica en las ciencias sociales y del comportamiento.

\section{3) Modelo instruccional transcriptor}

Conforme se hacía más evidente que los cursos de estadística dirigidos a presentar los modelos matemáticos en su carácter demostrativo o procedimental no eran suficientes para establecer en el alumno competencias de investigación, surgió otro modelo de enseñanza de la mano de libros de textos muy populares y que fueron los primeros en establecer un diálogo entre las abstracciones de los conceptos matemáticos y la metodología. En ellos se transcriben de manera metodológica los conceptos formales de la estadística, adaptándolos a los conceptos utilizados en las ciencias sociales y del comportamiento.

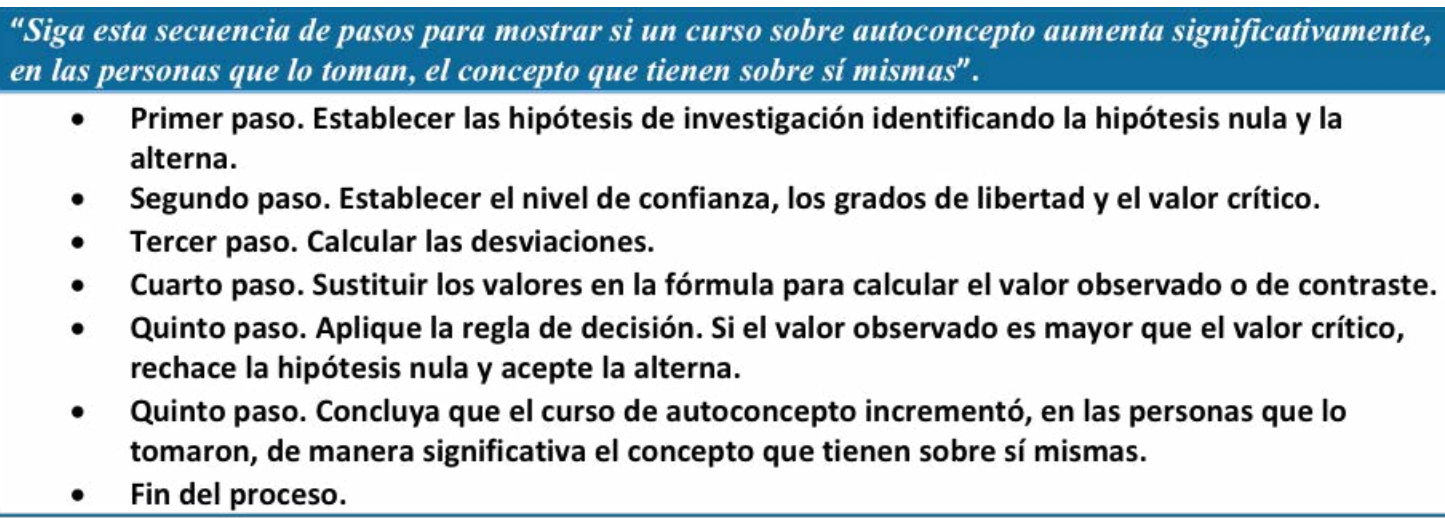

- Primer paso. Establecer las hipótesis de investigación identificando la hipótesis nula y la alterna.

- Segundo paso. Establecer el nivel de confianza, los grados de libertad y el valor crítico.

- Tercer paso. Calcular las desviaciones.

- Cuarto paso. Sustituir los valores en la fórmula para calcular el valor observado o de contraste.

- Quinto paso. Aplique la regla de decisión. Si el valor observado es mayor que el valor crítico, rechace la hipótesis nula y acepte la alterna.

- Quinto paso. Concluya que el curso de autoconcepto incrementó, en las personas que lo tomaron, de manera significativa el concepto que tienen sobre sí mismas.

- $\quad$ Fin del proceso.

Figura 1.

Ejemplo de la clase de ejercicios desde el modelo instruccional procedimental.

pocas asignaturas universitarias enfocadas a la iniciación temprana en investigación aún utilizan una didáctica enfocada en enseñar paso a paso el cálculo de las técnicas estadísticas. Todavía se puede leer en los libros de texto de reciente publicación, y retumban en las aulas universitarias, los ecos de la instrucción que se indica en la figura 1.

Como se muestra en el ejemplo de la figura 1, tomado de uno de los tantos libros que utiliza esta línea editorial, el principio básico de enfocarse en enseñar habilidades de investigación para que el estudiante desarrolle ejercicio tras ejercicio, característica definitoria de la tendencia demostrativa, aún es el mismo. La variante es que, en lugar de dirigir al estudiante a demostrar los teoremas matemáticos, la enseñanza se
El modelo instruccional transcriptor de iniciación temprana en investigación impactó de modo significativo en la enseñanza de la estadística, porque hizo evidente que no es suficiente abordar la teoría matemática que subyace a las herramientas de análisis cuantitativos de datos para establecer competencias de investigación en el estudiante, y en ocasiones puede ser hasta perjudicial, como lo describen Zapata-Cardona y González-Gómez (2017), por lo que su enseñanza se debe acompañar de los aspectos metodológicos de la manera en que los datos fueron recolectados; esto es, ligarlos a un contexto metodológico de investigación.

El esquema de enseñanza del modelo instruccional transcriptor es muy parecido al del apartado anterior; la 
única diferencia es que se adereza, hablando de manera metodológica, de una serie de definiciones de conceptos relacionados con la investigación empírica, pero que en esencia aún se mantiene una organización en la que se da prioridad a enseñar los conceptos lógico-matemáticos de las técnicas de investigación que se presentan en los libros y que se extrapolan a los salones de clase. para hacer los cálculos, sin dejar de mencionar que las salidas de los resultados de la computadora arrojan tales o cuales de datos. Las actividades didácticas en la clase consisten básicamente en una especie de recitativo donde los alumnos deben mencionar de memoria la definición de la técnica estadística y las salidas que arroja el software utilizado, en caso de tener la fortuna de poder

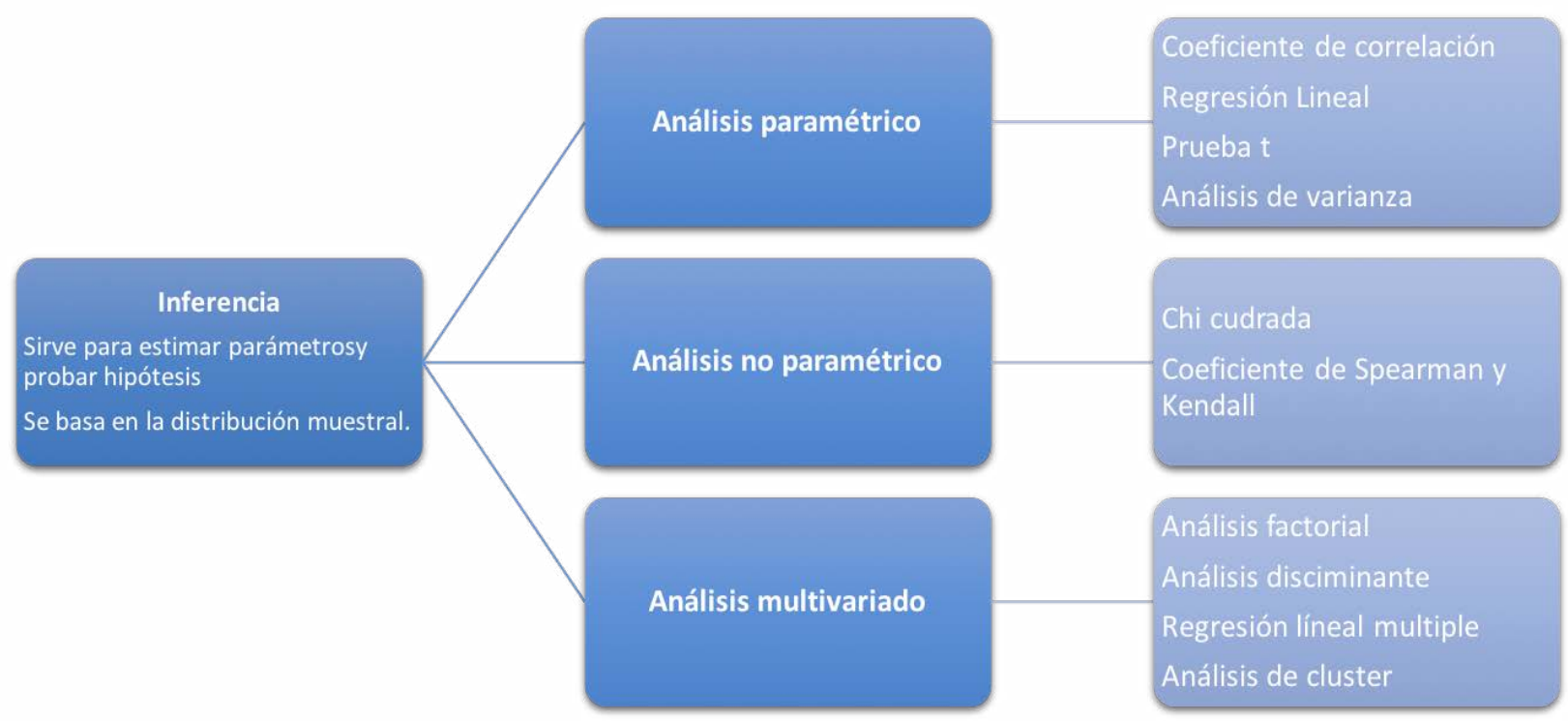

Figura 2.

Desglose de las trayectorias de la inferencia estadística.

La única diferencia es que ahora la mayoría de las veces se crea un diagrama que sirve de ruta de trabajo para tomar la decisión del uso de una determinada técnica estadística para aplicarse en una supuesta investigación. En ese tiempo empezaron a aparecer en los libros de texto esquemas como el que se muestra en la figura 2.

Una variante sencilla de este modelo fue reducir la enseñanza del proceso de investigación científica a una serie de preguntas que se responden en términos de definiciones - a la manera de un diccionario_-; se parte de una pregunta a la que se da una definición de lo que se cuestiona. Los libros que seguían esta línea editorial se asemejaban a un vademécum, porque son tratados que contienen sólo nociones de la información fundamental de lo que constituye la investigación científica. Se hacen preguntas como la que se plantea en el figura 3.

Y sin mayor preámbulo se pasa a desarrollar un ejemplo que incluye la hipótesis alterna; la mayoría de estos libros recomiendan utilizar un paquete estadístico acceder a un laboratorio de cómputo que identifique los diferentes grupos de resultados que produce la ejecución del comando correspondiente al análisis estadístico que se esté desarrollando.

El libro que vino a refrescar el modelo instruccional procedimental $-\mathrm{y}$ que en cierto sentido revolucionó la enseñanza de la investigación alejando el modelo de catequización en que cayó la mayoría de los libros que marcaron la tendencia transcriptora, y que aún es uno de los mejores que se ha escrito acerca de la manera de hacer y enseñar el proceso de la investigación científica- es el de Fred Kerlinger (1975), que como se desprende del subtítulo y se declara en el prólogo, se enfoca en asuntos conceptuales y técnicos abstractos, haciendo la aclaración de que éstos no se entenderán cabalmente sin la comprensión del propósito básico y la naturaleza de la investigación científica.

En esta etapa aparecieron otros libros de texto que pretendían vincular los modelos matemáticos con la inves- 
tigación, teniendo como punto de referencia la metodología científica e ir más allá de sólo traducir los conceptos formales, sobre todo de la estadística, en variables relacionadas con las ciencias sociales y del comportamiento, como la tradición en una época. El libro Métodos cuantitativos en Psicología, publicado en 1992, fue uno de esos blicación de ese libro surgió la idea de hacer una adecuación al modelo educativo del plan de estudios de Psicología que se impartía en la Facultad de Estudios Superiores Iztacala de la UNAM y fusionar las áreas de metodología y métodos cuantitativos que eran las encargadas de formar en el estudiante competencias rela-

“¿Qué es el análisis de varianza unidireccional o de un factor? (ANOVA one-way)”.
- Es una prueba estadística para analizar si más de dos grupos difieren de manera significativa
entre sí en cuanto a sus medias y varianzas. La prueba $t$ se aplica para dos grupos y el análisis
de varianza unidireccional se usa para tres, cuatro o más grupos. Aunque también se puede
utilizar con dos grupos.
- Hipótesis: de diferencia entre más de dos grupos. La hipótesis de investigación propone que
los grupos difieren significativamente entre sí y la hipótesis nula propone que los grupos no
difieren significativamente.
- Variables: una variable independiente y una variable dependiente.
Nivel de medición de las variables: la variable independiente es categórica y la dependiente es
por intervalos o razón.

Figura 3.

Modelo instruccional de preguntas y respuestas. esfuerzos porque además de estar enfocado en una disciplina en particular, su subtítulo (Un enfoque metodológico) declaraba con claridad su intención de considerar los aspectos conceptuales y formales del análisis cuantitativo desde una perspectiva metodológica teniendo como punto de referencia la investigación psicológica.

Con esta línea editorial ya se vislumbraba la necesidad de crear un modelo educativo dirigido a establecer competencias de investigación en el estudiante que consideraran no sólo la enseñanza de los conceptos lógicos-matemáticos de las herramientas de investigación, sino también que incorporaran los aspectos metodológicos. La razón de esta integración partía de la idea original del libro de Kerlinger, respecto a que no es posible hablar de una técnica estadística para analizar determinados datos cuantitativos sin haber adquirido determinada capacidad metodológica relacionada con la investigación científica. Un acierto más de esa obra es Ilamar la atención en la necesidad que tienen las ciencias sociales y del comportamiento de interpretar cualquier resultado en función del tipo de diseño y los procedimientos utilizados para reunir la base empírica en que se sustenta el conocimiento que generan dichas ciencias.

Esta variante en los libros de texto hizo que, en el plano formativo del estudiante, surgiera la necesidad de construir un nuevo modelo educativo que integrara los aspectos metodológicos, procedimentales y cuantitativos que comprenden el proceso de investigación científica en un espacio curricular único que abordara estos aspectos de manera holística. A inicios de la década de los noventa del siglo pasado, como resultado de la pu- cionadas con el diseño de investigaciones científicas y de análisis estadísticos. La iniciativa no cristalizó porque el clima académico y político que se vivía en esa época originó una disyuntiva que nunca se superó, ya que se presentó la iniciativa como una dicotomía, en el sentido de quién se fusiona con quién; es decir, el área de metodología con la de métodos cuantitativos o viceversa. Poco importó en ese momento la idea de generar un modelo educativo, que en su momento hubiera sido innovador, porque estaría más apegado a la manera en que se desarrolla el proceso de investigación científica integrando en un solo espacio curricular los asuntos conceptuales y técnicos con los propósitos y las competencias básicas que capaciten al estudiante para efectuar investigaciones en su disciplina de adscripción.

Con el surgimiento del modelo instruccional transcriptivo se establecieron las bases para, de manera temprana, iniciar al estudiante en investigación ayudándolo, ya sea mediante la producción de libros o cambiando su currículo formativo, para que entendiera la naturaleza fundamental que tienen tanto los conceptos lógicos-matemáticos y la metodología en el enfoque científico en la solución de los problemas que aquejan a nuestra sociedad. Un hecho histórico importante en los inicios de esta tendencia fue la aparición de las computadoras personales con su potencial didáctico en la enseñanza de competencias de investigación, lo que cambiaría de modo radical la línea editorial de los libros de texto y la manera en que se enseñaba a los estudiantes a investigar, originando el surgimiento de una cultura digital y 
una nueva tendencia en el establecimiento de competencias de investigación científica.

4) Modelo instruccional computacional (o la "cultura digital del simple o doble asterisco")

Como se refirió en el párrafo anterior, con el advenimiento de la computadora personal comenzó a gestarse una cultura digital en la sociedad que influyó de manera decisiva en la línea editorial de los libros de texto y en los espacios curriculares dedicados a formar al estudiante en investigación científica. Esta situación dio origen a la aparición de un nuevo modelo instruccional que podría denominarse "computacional".

Este modelo de iniciación temprana en investigación (ver figura 4) se caracteriza por el uso de un tipo de software que hace los cálculos de modo más rápido y exacto. Su adopción propició que en los cursos y en los libros de texto se dedicara más tiempo y espacio a

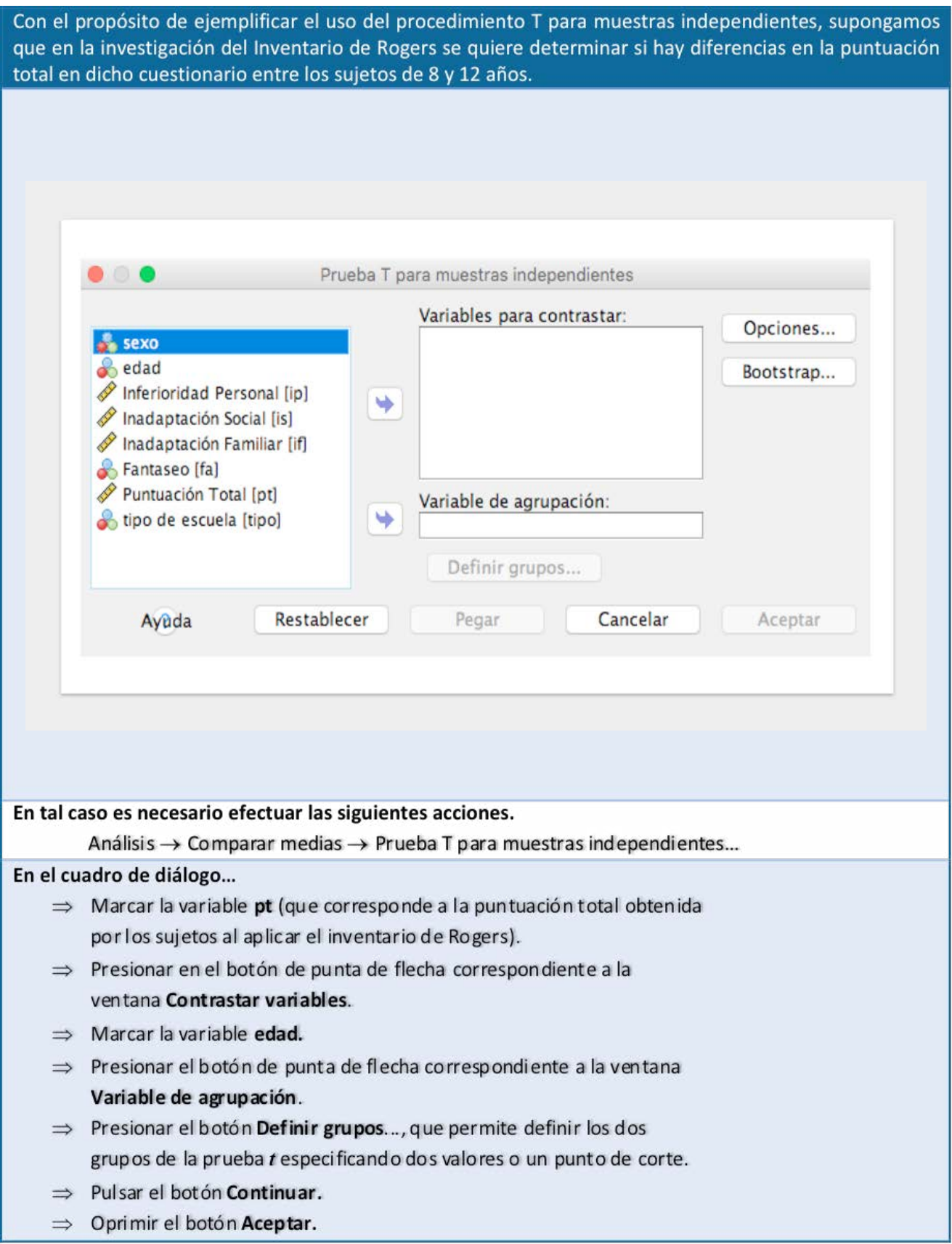

Figura 4.

Secuencia de enseñanza de en un modelo instruccional computacional.

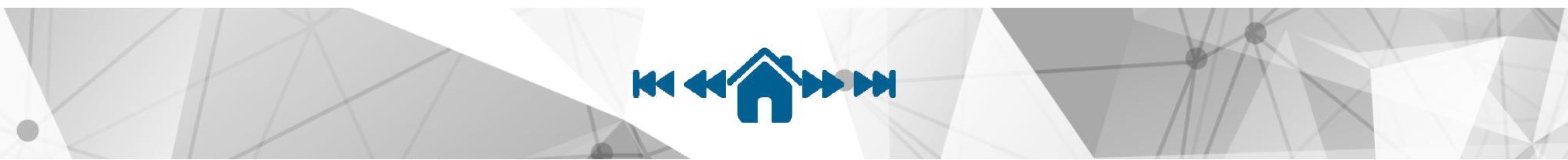


aprender los algoritmos computacionales o las secuencias de comandos con que se obtenían los resultados de la aplicación de una determinada técnica estadística.

Esta situación marcó el regreso de la tendencia demostrativa y se retomó su esencia, haciendo irrelevante la metodología y los procedimientos utilizados para recopilar la información en los esfuerzos de iniciar de manera temprana al estudiante en la investigación. De nuevo se excluyó del análisis el contexto que envuelve a la información recabada enfocando la atención en la secuencia para desarrollar un determinado comando. Las engorrosas y desconcertantes fórmulas del modelo instruccional demostrativo se sustituyeron por enseñar la interfaz con que se obtienen los resultados al aplicar una determinada técnica estadística. El proceso de investigación científica se revestió otra vez con un esmalte de azúcar grandilocuente de manejo de paquetes estadísticos, pero vacíos de contenido metodológico y aplicativo de los resultados obtenidos al ejecutar el algoritmo computacional.

En algunos círculos de científicos esta tendencia fue interpretada como un signo apocalíptico que marcaba el inicio de la desaparición en las universidades de las áreas académicas encargadas de enseñar los aspectos cuantitativos de la investigación científica y declararon la extinción de los profesores de estadística, con la premisa de que las computadoras ya hacían los cálculos estadísticos de toda investigación cuantitativa.

Esta perspectiva maniquea fue producto de la creencia que se tenía de que los cursos de estadística y los libros en que se basaban sólo se dedicaban al cálculo del resultado final de la aplicación de una técnica estadística, como lo hace el modelo procedimental que se analizó. Si bien algunos cursos y libros sólo se enfocaban a eso, había ya una tendencia en la didáctica de los cursos y en la línea editorial de los libros de texto de que los resultados de cualquier investigación dependen sobre todo de cómo se obtuvieron los datos y la metodología que se utilizó para registrarlos.

El uso de software ha aportado beneficios para la formación inicial de competencias de investigación en el estudiante, al sustituir la formulación de problemas por el uso de algoritmos computacionales; sin embargo se ha caído en una simplificación grotesca en la interpretación de los resultados porque ahora lo importante para el estudiante y en lo único que atiende, y en ocasiones hasta los investigadores, es que en las salidas que proporciona la aplicación de un software estadístico aparezcan el reverenciado asterisco, y si son dos mejor, que es la manera en que el software indica haber encontrado una diferencia estadísticamente significativa, o que una variable explica de modo satisfactorio los efectos observados en otra variable. Esta situación se ha denominado "cultura digital del simple o doble asterisco", porque la mayoría de los softwares colocan un asterisco cuando el resultado es estadísticamente significativo con una $\alpha$ de contraste igual a 0.05, y dos asteriscos cuando lo es a una $\alpha$ de contraste de 0.01. Una muestra de este tipo de cultura de "investigación" se encuentra en Hernández-Sampieri, Fernández-Collado y Baptista-Lucio (2014: 305): “[...] programas como IBM SPSS $®$ presentan los coeficientes de correlación en una tabla, donde las filas o columnas son las variables asociadas y se señala con asterisco(s) el nivel de significancia: un asterisco $(*)$ implica que el coeficiente es significativo al nivel del 0.05 y dos asteriscos ${ }^{(*)}$ que es significativo al nivel del $0.01^{\prime \prime}$.

Esta conducta irreflexiva que desvía la atención hacia el manejo del software y fijarse sólo en las salidas que éste produce, en ocasiones ha derivado en errores descomunales de lo que significa haber encontrado uno o dos asteriscos cuando se pasan por alto los supuestos teóricos de los que parten la investigación y el modelo matemático usado para poner a prueba la hipótesis o las hipótesis. Para entender la relevancia de los resultados encontrados no es suficiente con interpretar de modo adecuado la significancia estadística de los datos, sino conocer de dónde provienen y qué efecto tienen en la reformulación de las ideas y las direcciones futuras de la investigación (Casas-Rosal, Caridad y Ocerín, Núñez-Tabales y León-Mantero, 2019)

Por ejemplo, cada semestre - en el curso de ecuaciones estructurales que se imparte en el doctorado en Psicología de la UNAM - se dedica de una a dos clases a esclarecer que cuando se elabora un modelo de ecuaciones estructurales para explicar las relaciones causales que hay entre diferentes fenómenos de acuerdo con un modelo hipotetizado lo mejor es no encontrar ningún asterisco cuando se compara lo teórico con lo observado. Esta situación se debe a que lo que se está probando es un modelo de ajuste, no un modelo de diferencias como la t o el ANOVA; lo que se busca es qué tanto el modelo hipotetizado explica la base empírica recolectada al hacer la investigación. En este sentido, en ecuaciones estructurales el interés radica en no rechazar la hipótesis nula para garantizar que el modelo propuesto se ajusta de manera adecuada a los datos (Manzano-Patiño y Zamora-Muñoz, 2009). Esto es, que el modelo teórico planteado por el investigador explique lo más posible la realidad observada; su representación simbólica establece que: 
Donde:

$$
\Sigma=\Sigma(\boldsymbol{\theta})
$$

$\Sigma$ es la matriz de la covarianza de la población.

$\Sigma(\boldsymbol{\theta})$ es la matriz de covarianza que establece el modelo.

De manera que cuando la igualdad se mantiene, el modelo se ajusta a la base empírica encontrada al hacer la investigación, es decir, el modelo explica de manera significativa a los datos.

Esta situación describe cómo el uso irreflexivo de software mutila el proceso de investigación, coartando las posibilidades para el desarrollo del pensamiento creativo en el estudiante al carecer de una enseñanza orientada a la resolución de un problema, y sólo centrarla en la aplicación de un algoritmo computacional siguiendo los pasos que indica la interfaz de un software.

En ocasiones el uso irreflexivo ha sido de tal magnitud que hasta en artículos de prestigiados investigadores publicados en revista científicas reportan que el promedio del sexo en la muestra estudiada fue de 1.73, cuando en realidad este dato es producto de haber obtenido la información de una pregunta en la encuesta que rotulaba con 1 a los hombres y con 2 a las mujeres; obvio que el indicador más relevante en esta clase de preguntas no es el promedio, sino el índice de hombres y mujeres que constituyeron la muestra, aunque el algoritmo computacional haya calculado e informado del promedio de esa pregunta nominativa, porque como decía Lord (1953), "los números no saben de dónde vienen". Una mejor alternativa para evitar el uso irreflexivo del software es invertir el tiempo de formación del estudiante enseñándole destrezas que le permitan identificar e interpretar los indicadores cuantitativos más importantes de acuerdo con el objetivo de investigación en el mar de resultados que por lo general proporciona el software en la ejecución de un comando, que por lo común es más de una página impresa tamaño carta.

Una variante del modelo instruccional computacional es el uso de simuladores para recrear un determinado proceso estadístico; si bien esto permite que el estudiante conozca más a detalle dicho proceso, aún es necesario capacitarlo en investigación para que construya conocimiento científico. La simulación de procesos sólo le permite conocer a detalle el modelo matemático subyacente en el cálculo estadístico, pero no le permite avanzar en la comprensión del contexto, mientras que la investigación lo capacita en la creación de conocimiento científico al usar la estadística como un medio.

El modelo instruccional computacional revolucionó la manera de establecer competencias iniciales de investigación en el estudiante de pregrado en aspectos didácticos y de contenido. En el primer caso cambió de manera radical las actividades didácticas de la clase, porque el tiempo que se dedicaba en calcular manualmente determinada técnica estadística se eliminó al hacerlo por medio de un software. Por ejemplo, no fue necesario que el estudiante se involucrara en el cálculo de la inversa de una matriz; se podía hacer con la computadora, cuando antes se le pedía al estudiante que lo hiciera por diferentes métodos y que eligiera el de su preferencia, fuera éste por el de Gauss-Jordan, determinantes, cofactores, etcétera. En el segundo caso fue posible incorporar más contenido temático relacionado con las cuestiones metodológicas de la investigación y sus implicaciones en el conocimiento que generan las ciencias sociales y del comportamiento. De esta manera se amplió el horizonte para incorporar el contexto específico de donde provenía la base empírica para desarrollar determinado cálculo estadístico.

\section{5) Modelo instruccional integral}

En los últimos años ha surgido de manera paulatina un modelo instruccional en la enseñanza de las ciencias sociales y del comportamiento abocado a diseñar espacios curriculares que sean integrales en sustitución de los cursos tradicionales de estadística, matemáticas y algebra, en donde se programen actividades relacionadas con la reflexión teórica, el uso de diversas metodologías para recolectar datos, la aplicación de modelos matemáticos, estadísticos y cualitativos para analizar de modo minucioso la información recabada y el desarroIlo del pensamiento analítico enfocado a verificar - ya sea confirmar o refutar- hipótesis provisionales.

Estos espacios curriculares de iniciación temprana en investigación en las ciencias sociales y del comportamiento se alejan del modelo de enseñanza de las ciencias formales dirigidas a demostrar o probar sus enunciados. Este alejamiento se debe a que como ciencias fácticas su campo de acción no sólo es explicar los fenómenos, sino también proponer alternativas de solución a los problemas socialmente relevantes. En consecuencia, dichas ciencias invierten una gran cantidad de esfuerzos en el campo de la investigación empírica con la finalidad de explicar los fenómenos. Asimismo, recurren a los métodos cuantitativos y cualitativos para analizar sus fenómenos; debido a esto, la mejor manera de formar competencias de investigación en el alumno es adoptar un modelo instruccional integral en las que las abstracciones matemáticas y epistemológicas sirvan como herramientas para fundamentar las interpretaciones de la base empírica recolectada al hacer el estudio. 
Desde un modelo instruccional integral las interpretaciones requieren muchas habilidades no matemáticas; menos aún necesitan las matemáticas utilizadas para derivar fórmulas; en todo caso serán necesarias habilidades computacionales que permitan obtener evidencias cuantitativas rápidas y precisas que faciliten la comprensión de lo que se está estudiando (GAISE-College-Report, 2016).

Este viraje implica motivar y capacitar al estudiante en cuestiones teóricas, técnicas y prácticas del proceso de investigación en las ciencias sociales y del comportamiento, para desarrollar en él la creatividad y la capacidad de innovación que le permitan descubrir, consolidar y refinar los conocimientos que hoy se tienen en las distintas disciplinas que conforman las ciencias sociales y del comportamiento. Además, el estudiante tendrá la oportunidad de adquirir experiencia en la que se integre lo abstracto de las matemáticas con lo concreto del contexto que envuelve a la investigación científica. Esta experiencia le será útil no sólo al estudiante interesado en continuar con estudios de posgrado, sino también al que quiera ingresar de manera directa al campo laboral cuando finalice su carrera, ya que cada vez hay más puestos de trabajo de profesionales que tengan competencias relacionadas con la investigación científica (Carter, Brown y Simpson, 2017; Jones, 2019; Songsore y White, 2018).

Una integración real requiere un diseño curricular que organice los contenidos de los cursos de estadística, álgebra y matemáticas, o sus variantes de métodos cuantitativos en dos grandes ejes de competencias científicas; uno que enfocado a establecer competencias en el alumno que le permitan elaborar explicaciones científicas a partir de los hallazgos reportados en investigaciones publicadas en revistas científicas, y el otro que lo capacite en el diseño de investigaciones científicas que le permitan contrastar las diversas explicaciones que se ofrecen de un mismo fenómeno, o mostrar la efectividad de una determinada estrategia de intervención en la solución de problemas socialmente relevantes.

El primer eje de este modelo instruccional integral de enseñanza podría abocarse a establecer en el estudiante competencias y habilidades para diseñar, aplicar y evaluar investigaciones orientadas hacia la formulación exploratoria de teorías explicativas de las ciencias sociales y del comportamiento; esto es, hacia la búsqueda de enunciados lógicos explicativos de las relaciones observadas. Con esto se pretende que el estudiante adquiriera la capacidad para establecer vínculos lógicos, al partir de evidencias cuantitativas y cualitativas entre distintas proposiciones teóricas, con las cuales estaría en posibilidad de generar, desde su perspectiva, argumentos que unifiquen los elementos que componen las diferentes teorías.
El segundo eje estaría diseñado a formar en el estudiante competencias que lo capaciten para poner a prueba las teorías que hay en las ciencias sociales y del comportamiento. Razón por la cual los contenidos curriculares podrían enfocarse hacia el diseño, aplicación y evaluación de investigaciones científicas cuyos resultados le permitan seleccionar entre diferentes formulaciones teóricas alternativas, con el objetivo de llegar a una clase nueva y diferente de conocimiento científico.

Al adoptar el modelo instruccional integral en la iniciación temprana en investigación, la planeación didáctica de los cursos debe considerar que la enseñanza de un modelo matemático, estadístico o cualitativo esté siempre acompañada de las implicaciones que su uso tiene en la explicación de los fenómenos propios de las ciencias sociales y del comportamiento. Una manera de hacerlo es considerar, por un lado, la contigüidad cuantitativa y cualitativa de los fenómenos de las ciencias sociales y del comportamiento, y por otro lado el método de contigüidad funcional, para que el estudiante integre distintos procesos, tanto en su dimensión cuantitativa y cualitativa, así como funcional, no obstante que todas sean el resultado de situaciones, contextos o momentos históricos diferentes.

Esta nueva organización tendría la ventaja de incluir criterios donde el alumno evalúe de manera constante el conocimiento descubierto, consolidado y refinado al hacer en todo momento referencia a la pertinencia del conocimiento obtenido en las investigaciones que haga, en relación con los conceptos utilizados y en la base empírica obtenida.

Uno de dichos criterios sería la validez del conocimiento obtenido buscando que no tenga contradicciones, como lo establece el paradigma formal o lógico deductivo. Uno más sería que los conocimientos generados por el estudiante expliquen y predigan los procesos que esté examinando, a la manera como lo establecen los paradigmas hipotético deductivo y analítico inductivo. Otros criterios podrían ser la confiabilidad, generabilidad y explicabilidad que permitiría evaluar las actividades de investigación que el estudiante desarrolle, encaminadas a descubrir, consolidar, refinar y aplicar el conocimiento propio de las ciencias sociales y del comportamiento.

Además de estos criterios, la planeación didáctica del modelo instruccional integral de iniciación temprana a la investigación requiere que los espacios curriculares de iniciación temprana en investigación contengan evidencia empírica que provenga del mundo real y en la que se dé prioridad al establecimiento de competencias flexibles que permitan al estudiante resolver los problemas que se presentan al hacer la investigación. De igual 
manera es necesario que los problemas que se le planteen sean derivados de un contexto que le sea significativo, y que a su vez la pregunta de investigación le sea motivadora. Otro aspecto que es necesario considerar es la inclusión de actividades que fomenten la síntesis de teoría, metodología y computación, y que desemboquen en la elaboración del reporte de los resultados encontrados al efectuar la investigación.

\section{Discusión}

En el marco de la iniciación temprana en investigación, el análisis desarrollado en este artículo abarcó lo que se conoce como "investigación formativa", que considera a la investigación misma como instrumento del proceso de enseñanza-aprendizaje, así como la formación para la investigación, donde se incluyen —según varios autores- las acciones que permiten al estudiante adquirir competencias para desempeñarse de modo adecuado en el sector académico o productivo desarrollando actividades profesionales asociadas directamente con la investigación científica, el desarrollo tecnológico y la innovación (Gómez-Restrepo, 2017; Guerra-Molina, 2017; Guerrero-Useda, 2007; Montoya y Peláez, 2013; Numa-Sanjuan y Márquez-Delgado, 2019).

La enseñanza universitaria temprana en investigación ha seguido varios caminos; su elección ha dependido de la tendencia en boga y de la decisión de cuál es el sello que la universidad pretende imprimir en sus egresados. Las asignaturas de estadística han sido los espacios curriculares por excelencia para enseñar al estudiante de pregrado algunas de las competencias relacionadas con la investigación científica. En el currículo de algunas disciplinas de las ciencias sociales y del comportamiento en ocasiones se programan asignaturas de matemáticas, álgebra y calculo, consideradas como prerrequisitos para incursionar en el análisis cuantitativo de las investigaciones mediante la estadística. Sin bien estas asignaturas son esfuerzos encomiables dirigidos a formar en el estudiante competencias básicas para investigación, la mayoría prestan muy poca atención a incluir contenidos temáticos que fortalezcan los objetivos educativos de iniciar de modo temprano a la investigación; más bien son asignaturas dedicadas a mostrar los algoritmos estadísticos, así como la manera de calcularlos ya sea manualmente o por medio del uso de algún software (Strayer, Gerstenschlager, Green, McCormick, MaDaniel y Rowell, 2019). Además es común que sean consideradas por la comunidad estudiantil como asignaturas con muy poca relevancia práctica en la vida profesional futura (Songsore y White, 2018).
Es muy posible que esta falta de correspondencia sea producto del modelo instruccional que se usa en las universidades para formar tempranamente competencias de investigación, lo que a su vez origina una percepción negativa en el estudiante respecto a la relevancia de los contenidos que se estudian en esas asignaturas (Immekus, 2019). Esta percepción es entendible porque es común que en las asignaturas se invierta gran parte del tiempo en demostrar los algoritmos involucrados en los análisis, o en el mejor de los casos a su cálculo paso a paso, o mediante el uso de software computacional.

Los modelos instruccionales que de manera tradicional se han usado en las universidades son causantes de esta situación, y lo que es más grave en la poca efectividad que han mostrado estas asignaturas para iniciar tempranamente al estudiante en investigación. Desde el modelo demostrativo, procedimental, transcriptivo y hasta el computacional, ninguno de ellos está enfocado a proporcionar al estudiante las habilidades necesarias para experimentar con datos, encontrando sus patrones específicos y que de esta manera él haga sus propias preguntas para responderlas por medio del desarrollo de una investigación en la que se involucre en la obtención de las evidencias empíricas que le permitan responder a los supuestos que él mismo se plantee. La debilidad de estos modelos instruccionales radica en que al carecer de evidencias empíricas obtenidas en contextos reales impide al estudiante relacionar los conceptos abstractos matemáticos, epistemológicos y estadísticos con la realidad, haciéndolos para el estudiante poco atractivos y alimentar la creencia de su poca o nula utilidad en el ejercicio de su profesión.

Lo más grave de los modelos instruccionales dirigidos a iniciar tempranamente a la investigación es que los primeros cuatro no encauzan al estudiante a evaluar de modo crítico los estudios empíricos ni a generar preguntas de investigación comprobables, menos aún a seleccionar y establecer estrategias metodológicas que produzcan hallazgos empíricos que enriquezcan la teoría o que al menos bosquejen una factible solución al problema investigado. Son modelos que sólo enseñan una parte de lo que comprende el proceso de investigación científica; el demostrativo a revelar la lógica matemática que sustenta el algoritmo de análisis; el procedimental a mostrar paso a paso la manera de obtener el valor de contraste; el transcriptivo a traducir los conceptos matemáticos en el ámbito disciplinar que haga uso de esos conceptos, y el computacional enseña sólo la manera de calcular los valores de contraste utilizando herramientas automatizadas de software.

Si en realidad se pretende impulsar en las universidades la iniciación temprana en investigación, es nece- 
sario incorporar en la enseñanza de cualquier disciplina de las ciencias sociales y del comportamiento un modelo instruccional integral en el cual se diseñen estrategias de aprendizaje donde se conjugue la reflexión teórica, el uso de metodologías variadas, se apliquen procedimientos cualitativos y cuantitativos para analizar la base empírica y que desarrollen el pensamiento analítico que contraste las hipótesis de investigación y que se deriven sugerencias de cambio que mejoren las condiciones actuales.

El impulso de esta iniciativa necesita, por un lado, reorganizar los espacios curriculares asignados de modo tradicional a impartir asignaturas relacionadas con las ciencias formales (como el álgebra, las matemáticas y su variante la estadística pura), y por otro lado utilizar esos espacios para formar competencias que capaciten al estudiante en el diseño de investigaciones científicas en ambientes reales. De no hacer esto tempranamente cuando el estudiante inicie sus estudios de pregrado se impedirá que las ciencias sociales y del comportamiento contribuyan a la explicación, comprensión y solución de problemas socialmente relevantes, así como a obstaculizar que el estudiante se convierta en un ciudadano informado más activo y crítico que se inserte libre y rápidamente al campo laboral, o que inicie sus estudios de posgrado transparentemente y sin tanta dificultad.

\section{Conclusiones}

En la sociedad de hoy se incrementa cada vez más la necesidad de ampliar la fuerza laboral técnicamente calificada en investigación, no sólo en la denominada en la introducción de este artículo como "erudita", que es la que cultivan los círculos de científicos especializados, sino también en la que se hace en los sectores público y privado dirigida a evaluar estrategias de intervención para conocer, entre muchas cosas, el impacto de programas y políticas sociales instrumentadas por organizaciones gubernamentales, de la sociedad civil, empresariales, etcétera (Williams, Payne, Hodgkinson y Poade, 2008).

Ante este escenario es impostergable estimular la iniciación temprana en investigación desde el pregrado para contribuir de modo positivo en la formación profesional del estudiante al capacitarlo en la integración teórica y reflexión analítica epistemológica, así como en la gestión de datos, computación, fundamentación matemática y estadística. La mejor manera de hacer esto es mediante la inserción del estudiante en escenarios reales que hagan atractiva la aplicación de los conceptos abstractos en investigaciones prácticas que los despojen del halo de misterio que acompaña a todo concepto abstracto derivado de la teoría (Roy, 2019).
Las habilidades y competencias que adquiera en esos contextos reales le serán útiles no sólo para ingresar a estudios de posgrado, sino también incrementará la posibilidad de insertarse a la población económicamente activa. En organizaciones públicas y privadas cada vez aumenta más la demanda de profesionales que tengan competencias en investigación y habilidades para tomar decisiones acertadas a partir del análisis de la información empírica que se disponga. En la sociedad actual la necesidad de este tipo de profesionales es muy grande; McKensey \& Company (2011) afirmaba que para 2018 habría una escasez de profesionales con habilidades de investigación, así como de investigadores, gerentes y analistas con conocimiento de análisis de Big Data y la manera de utilizarlo en la toma de decisiones efectivas respaldadas por principios científicos (Jones, 2019). Esta predicción se cumplió y ahora se puede ver en amplios sectores de la sociedad la necesidad de profesionales con competencias para investigar los más variados problemas que enfrenta la sociedad actual a través de Big Data.

Este escenario de oportunidad se amplía más al considerar que la investigación en las ciencias sociales y del comportamiento es un campo de conocimientos que abarca un nivel muy amplio de áreas de aplicación con un uso generalizado en la sociedad moderna. En el campo de conocimientos se incluyen las dimensiones cuantitativa y cualitativa que, al margen de sus diferencias y semejanzas, en ambas se trabaja con una base empírica de datos que para analizarla es necesario disponer de un sinnúmero de habilidades para interpretar y comprender los significados involucrados en el mar de información contenida en ella. Con esa base empírica se pretende descubrir nuevos conocimientos científicos, identificar modelos aplicados que permitan cambiar el rumbo de los acontecimientos para generar propuestas de prevención que respondan a problemas en la sociedad. Estas acciones requieren que el estudiante tenga un punto de vista teórico consistente, desarrolle un acercamiento metodológico riguroso, anclado en la realidad, y que combine con prudencia los trabajos básico y aplicado. Por tanto, para iniciar tempranamente al estudiante en investigación es indispensable proporcionarle las habilidades para experimentar con datos, encontrar sus propios modelos y hacer sus propias preguntas (Loy, Kuiper, y Chihara, 2019).

Si bien la mayoría de las investigaciones se desarroIla en las universidades e institutos de investigación, hay otros escenarios donde también se hacen y que responden a múltiples propósitos, en los cuales el egresado de pregrado puede ejercer su profesión. Uno de dichos escenarios es en el sector privado, en el que se tiene especial interés en las investigaciones de mercado porque éstas 
proporcionan información a las empresas, entre otras cosas, respecto a los hábitos de consumo de sus clientes, a partir de los cuales pueden establecer las diferentes políticas, objetivos, planes y estrategias más adecuadas que respondan a los intereses de consumo de su clientela. Las instituciones de gobierno también efectúan investigaciones en el contexto de las ciencias sociales y del comportamiento, sobre todo para evaluar el impacto de los programas sociales que se instrumentan, derivado de las políticas que se establecen (como en los planes nacionales de desarrollo de los países, en los que se pretende materializar el principio de equidad e incrementar la eficiencia en el logro de las metas establecidas en dichos planes). Asimismo, la investigación de las ciencias sociales y del comportamiento se desarrolla en ámbitos como la justicia penal, el sector salud, el periodismo, la sociología, la psicología, la educación, la economía, etcétera.

Como es evidente por estas breves ideas, la investigación toma variadas formas y se aplica en muchas situaciones en el mundo real, y en todas ellas se enfoca a explicar, comprender y modificar los sucesos que conforman la estructura del mundo social. Esta tarea la efectúa combinando estudios explicativos, predictivos y humanísticos cuyo centro son las habilidades técnicas de investigación (Payne, Williams y Chamberlain, 2004).

En este mundo global es necesario cambiar de manera radical el modelo educativo para formar los futuros profesionales egresados de alguna disciplina de las ciencias sociales y del comportamiento. El principal cambio implica abandonar el enfoque tradicional de pretender establecer competencias básicas de investigación por medio de incluir asignaturas de estadística en el currículo de estudios de pregrado. En el transcurso de los años este enfoque ha mostrado su ineficacia, porque en lugar de fomentar dichas competencias básicas ha propiciado en el estudiante un sentimiento de rechazo, malestar y zozobra hacia los contenidos temáticos de esas asignaturas (Carter et al., 2017; Cobb y Moore, 1997; Songsore y White, 2018; Williams et al., 2008).

El giro implica adoptar un enfoque que se base en la investigación y que se utilicen datos del mundo real, lo que permitirá proporcionar al estudiante un conjunto de competencias aplicadas que podrá transferir de modo rápido y transparente a cualquier entorno laboral, donde los problemas rara vez se presentan a la manera como se hace en los libros de texto con demostraciones de algoritmos matemáticos, siguiendo procedimientos estándares para obtener el resultado, transcribiendo conceptos, o haciendo uso sólo de un software.

Por último, con este cambio de enfoque no sólo se beneficiará el estudiante, sino también los empleadores de los egresados de las ciencias sociales y del comportamiento del sector público, privado e independiente al incorporar a sus organizaciones la conciencia crítica y mentalidad analítica propias del pensamiento científico. El resultado sería formar un estudiante con herramientas de investigación que le facilite el tránsito a los estudios de posgrado, o que lo haga más competitivo en el mundo laboral. En este último caso se contribuiría a crear una población de graduados alfabetizados tempranamente en investigación listos para la fuerza laboral que impulsen políticas sociales, gubernamentales y empresariales basadas en evidencias empíricas ya sea de naturaleza cuantitativa o cualitativa.

En un siguiente artículo se presentarán la estructura y las actividades de enseñanza de un modelo educativo integral enfocado a iniciar tempranamente en investigación al estudiante de pregrado, donde los espacios curriculares que se usan de modo tradicional para impartir asignaturas de matemáticas, álgebra, estadística y metodología científica se transforman en una zona del plan de estudios de cualquier disciplina de las ciencias sociales y del comportamiento, en donde se fomenta la iniciación temprana a la investigación y el trabajo en equipo que propicia en el estudiante una constante actividad de reflexión al participar de manera activa en el descubrimiento, consolidación y refinamiento del conocimiento que se genera en las ciencias sociales y del comportamiento.

\section{Referencias}

Anderson, D. R., Sweeney, D. J., \& Williams, T. A. (1999). Estadística para administración y economía. México: CENGAGE Learning.

Baum, S., \& Steele, P. (2017). Who Goes to Graduate School and Who Succeeds? (Vol. null).

Blalock, H. M. (1978). Estadística social. México: Fondo e Cultura Económica.

Carter, J., Brown, M., \& Simpson, K. (2017). From the Classroom to the workplace: how social science students are doing data analysis for real. Statistics Education Research Journal, 16(1), 80-101.

Casas-Rosal, J. C., Caridad y Ocerín, J. M., Núñez-Tabales, J. M., \& León-Mantero, C. (2019). Teaching statistics through the Real Estate Data Analyzer software. Teaching Statistics, 41 (2), 58-64. doi: 10.1111/test.12183

Cobb, G. W., \& Moore, D. S. (1997). Mathematics, Statistics, and Teaching. The American Mathematical Monthly, 104 (9), 801-823. doi: $10.2307 / 2975286$

Cvetkovic-Vega, A., Inga-Berrospi, F., \& Mestas, C. A. (2017). Organizaciones científicas estudiantiles como semilleros de líderes y gestores de la investigación científica en el Perú: SOCIMEP. Acta Médica Peruana, 34(1), 70-71.

Daniel, W. W. (1977). Bioestadística : Base para el análisis de las ciencias de la salud México: LIMUSA-Noriega. 
Eudave Muñoz, D., Carvajal Ciprés, M., \& Páez, D. A. (2018). Importancia y utilidad de la estadística en profesiones del campo de las ciencias sociales. RECIE. Revista Electrónica Científica de Investigación Educativa, 4(1), 283-293.

GAISE-College-Report. (2016). Guidelines for Assessment and Instruction in Statistics Education College Report 2016. http://www.amstat.org/education/gaise.

Glass, G. V., \& Stanley, J. C. (1974). Métodos estadísticos aplicados a las ciencias sociales: Prentice Hall Hispanoamericana S. A.

Gómez-Restrepo, C. (2017). Semilleros de investigación: impulso a la innovación, el aprendizaje y la docencia. Universitas Médica, 58(1).

Guerra-Molina, R. (2017). ¿ Formación para la investigación o investigación formativa?. La investigación y la formación como pilar común de desarrollo. Revista Boletín Redipe, 6(1), 84-89.

Guerrero-Useda, M. E. (2007). Formación de habilidades para la investigación desde el pregrado. Acta colombiana de psicología, 10(2), 190-192.

Hernández-Sampieri, R., Fernández-Collado, C., \& BaptistaLucio, M. d. P. (2014). Metodología de la investigación. México: McGraw Hill.

Immekus, J. C. (2019). Flipping Statistics Courses in Graduate Education: Integration of Cognitive Psychology and Technology. Journal of Statistics Education, 27(2), 79-89. doi: 10.1080/10691898.2019.1629852

Jones, R. C. (2019). Statistical investigation measuring intelligence and creativity. Teaching Statistics, 41(1), 3640. doi: $10.1111 /$ test.12169

Kerlinger, F. (1975). Investigación del comportamiento. Técnica y metodo. México: NO SE.

Lord, F. M. (1953). On the Statistical Treatment of Football Numbers. American Psychologist, 8(12), 750-751. doi: $10.1037 / h 0063675$

Loy, A., Kuiper, S., \& Chihara, L. (2019). Supporting Data Science in the Statistics Curriculum. Journal of Statistics Education, 27(1), 2-11. doi: 10.1080/10691898.2018.1564638

MacGillivray, H. (2019). Statistics and data science are NOT branches of mathematics - or of any other discipline. Teaching Statistics, 41(2), 41-41. doi: 10.1111/test.12197

Manzano-Patiño, A., \& Zamora-Muñoz, S. (2009). Sistema de ecuaciones estructurales: una herramienta de investigación. México: Centro Nacional de Evaluación para la Educación Superior, A. C. .

McKensey\&Company. (2011). Big data: The next frontier for innovation, competition, and productivity. https:// bigdatawg.nist.gov/pdf/MGI big data full report.pdf

Montoya, J., \& Peláez, L. E. (2013). Investigación formativa e investigación en sentido estricto: una reflexión para diferenciar su aplicación en instituciones de educación superior. Entre Ciencia e ingeniería, 13, 20-25.

Nerlove, M., Grether, D. M., \& Carvalho, J. L. (1988). Análisisa de series temporales económicas. México: Fondo de Cultura
Económica.

Numa-Sanjuan, N., \& Márquez-Delgado, R. A. (2019). Los semilleros como espacios de investigación para el investigador novel. Propósitos y Representaciones, 7, 230239. doi: 10.20511/pyr2019.v7n1.289

Pardinas, F. (1969). Metodología y técnicas de la investigación en ciencias sociales. México: Siglo XXI.

Payne, G., Williams, M., \& Chamberlain, S. (2004). Methodological pluralism in British sociology. Sociology, 38(1), 153-163.

Rodríguez-Morales, A., Milanés-Gómez, R., \& Avila-Portuondo, A. M. (2016). La profesionalización una fuente para semilleros de investigación. Revista Conrado, 12(55), 83-89.

Rojek, C., \& Turner, B. (2000). Decorative Sociology: Towards a Critique of the Cultural Turn. The Sociological Review, 48(4), 629-648. doi: 10.1111/1467-954x.00236

Roy, S. (2019). Inferential statistics made fun: stories that boxes of '100 paper clips' can tell. Teaching Statistics, 41(1), 25-29. doi: $10.1111 /$ test.12168

Saavedra-Cantor, C. J., Antolínez-Figueroa, C., Puerto-Guerrero, A. H., Muñoz-Sánchez, A. I., \& Rubiano-Mesa, Y. L. (2015). Semilleros de investigación: desarrollos y desafíos para la formación en pregrado. Educación y educadores, 18(3), 391-407.

Silva-Rodriguez, A. (1992). Metodos cuantitativos en psicología. Un enfoque metodológico. México: Trillas.

Songsore, E., \& White, B. J. (2018). Students' perceptions of the future relevance of statistics afer completing an online introductory statistics course. Statistics Education Research Journal, 17(2), 120-140.

Spiegel, M. R. (1970). Estadística. México: MacGraw-Hill.

Spiegel, M. R. (1977). Probabilidad y estadística. México: MacGraw-Hill.

Strayer, J. F., Gerstenschlager, N. E., Green, L. B., McCormick, N., MaDaniel, S., \& Rowell, G. H. (2019). Toward a full(er) implement of active learning. Statistics Education Research Journal, 18(1), 63-82.

Tovar-Torres, C. (2018). Investigación formativa. Perspectivas(11). Villalba-Cuéllar, J. C., \& González-Serrano, A. (2017). La importancia de los semilleros de investigación. Revista Prolegómeno, 20(39), 9-10.

Wesbster, A. L. (2000). Estadística aplicada a los negocios y a la economía. México: MacGraw-Hill.

Williams, M., Payne, G., Hodgkinson, L., \& Poade, D. (2008). Does British Sociology Count? Sociology Students' Attitudes toward Quantitative Methods. SAGE, 42(5), 1003-1021.

Zapata-Cardona, L., \& González-Gómez, D. (2017). Imágenes de los profesores sobre la estadística y su enseñanza. Educación matemática, 29, 61-90.

Zapata-Peña, J., Ortiz-Puerto, A. C., Bautista-Rincón, R., DíazGonzález, J., Tinoco, L. A., \& Ayala-Cortés, C. (2014). Una experiencia sobre la conformación de semilleros de investigación en la formación inicial de profesores. Revista EDUCYT, 9, 1-9. 